\title{
Tratamiento del eccema de manos
}

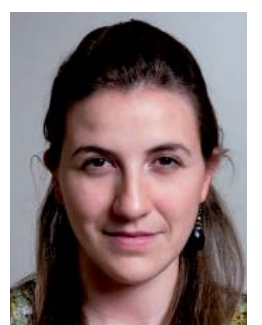

María Lucrecia Infante Hernando Dermatóloga. Unidad de Alergia Cutánea. Hospital de la Santa Creu i Sant Pau. Barcelona

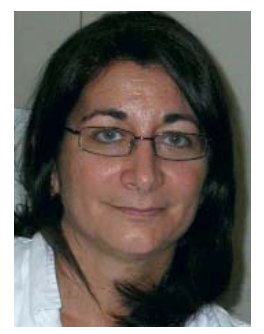

Esther Serra Baldrich Médico adjunto. Servicio de Dermatología. Unidad de Alergia Cutánea. Hospital de la Santa Creu i Sant Pau. Barcelona

\section{INTRODUCCIÓN}

Resulta complicado definir la entidad denominada eccema de manos ya que, si somos estrictos, existen muy diversas situaciones que pueden ser responsables de los diferentes eccemas que se localizan en las manos, como el eccema atópico, numular, hiperqueratósico y de contacto ${ }^{1}$. A pesar de ello, en general se habla del eccema de manos especialmente vinculado al contacto con agentes exógenos irritantes o alergénicos. Hay que esforzarse también por diferenciar el eccema de esta localización de otras dermatosis, como la psoriasis.

La prevalencia del eccema de las manos se sitúa aproximadamente en un $15 \%$ y su incidencia se ha asociado mayormente con el sexo femenino, la dermatitis atópica, la alergia de contacto y el trabajo en ambiente húmedo (fig. 1). El pico de edad en mujeres se sitúa sobre los 20 y los 30 años $^{2}$. Otro factor de riesgo en el

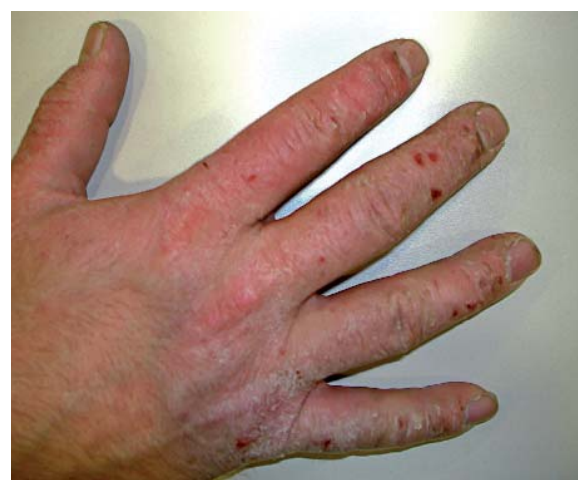

Figura 1. Eccema de manos. Se observan eritema, descamación, fisuras y vesículas rotas. desarrollo del eccema es la presencia de mutaciones en el gen de la filagrina, que altera la función de barrera cutánea.

La alergia al níquel es otro factor de riesgo para el eccema de manos, pero las intervenciones reguladoras sobre la exposición a este metal, así como cambios en el medio ambiente, han conseguido reducir esta asociación en algunos países².

La duración media del eccema de manos sobrepasa los 10 años, y el eccema de contacto irritativo es más prevalente que el atópico y el alérgico.

Sobre la persistencia del mismo, parece ser que una mayor extensión y un inicio precoz son factores importantes.

Veien et al. ${ }^{3}$ propusieron una clasificación del eccema de manos basada en tres aspectos:

1. Siempre que sea posible, realizar un diagnóstico etiológico (dermatitis de contacto alérgica, irritativa, atópica, etc.).

2. Diagnóstico morfológico de la dermatitis (eccema crónico fisurado, vesicular recurrente, palmar hiperqueratósico, pulpitis, interdigital, numular).

3. Identificación de la dinámica de la dermatitis.

Los estudios futuros en eccema de las manos sobre la población general se dirigirán en lo posible hacia la investigación genética y seguramente 
las estrategias terapéuticas y de prevención diferirán según los diferentes grupos de individuos.

\section{ASPECTOS PSICOSOCIOLABORALES}

El eccema de manos es una enfermedad episódica y crónica con un impacto negativo sobre la calidad de vida de los pacientes afectados ${ }^{4,5}$, comparable a los pacientes con dermatitis atópica y psoriasis $^{6}$. Es una entidad frecuente que se presenta con síntomas clínicos variados, desde cuadros muy leves hasta tan severos que pueden llevar a la baja laboral, cambio de ocupación o discapacidad permanente. Si tenemos en cuenta que el $50 \%$ de los casos son por exposición ocupacional, esta enfermedad representa un alto costo socioeconómico para el paciente y la sociedad ${ }^{6}$.

En un estudio multicéntrico de corte transversal realizado por Agner et al., se observó una correlación entre severidad del eccema de manos (Hand Eczema Severity Index, HECSI, tabla 1) y afecta- ción de la calidad de vida (Dermatoloy Life Quality Index, DLQI), con mayor afectación de la calidad de vida en cuadros más severos. Asimismo, no se encontraron diferencias significativas en el impacto sobre la calidad de vida entre mujeres y hombres, a pesar de la mayor afectación clínica de estos últimos ${ }^{5}$.

\section{MEDIDAS PREVENTIVAS Y EDUCACIÓN DEL PACIENTE CON ECCEMA DE MANOS}

En la prevención de nuevos episodios, se deben contemplar dos aspectos: eliminación de los agentes de contacto, si es posible, o las condiciones de trabajo favorecedoras, como el exceso de humedad y el uso de guantes ocluidos durante mucho tiempo; mejora de la función de barrera cutánea con guantes de algodón y aplicación de cremas emolientes.

Tabla 1. Índice de severidad del eccema de manos (Hand Eczema Severity Index, HECSI) ${ }^{6}$.

\begin{tabular}{|c|c|c|c|c|c|}
\hline \multirow[b]{2}{*}{$\begin{array}{l}\text { Signos clínicos/ } \\
\text { extensión }\end{array}$} & \multicolumn{5}{|c|}{ Localización } \\
\hline & $\begin{array}{l}\text { Pulpejos } \\
\text { de dedos }\end{array}$ & $\begin{array}{c}\text { Dedos (sin } \\
\text { pulpejos) }\end{array}$ & Palmas & $\begin{array}{c}\text { Dorso } \\
\text { de manos }\end{array}$ & Muñecas \\
\hline $\begin{array}{l}\mathbf{0}=\text { ausente } \\
\mathbf{1}=\text { leve } \\
\mathbf{2}=\text { moderada } \\
\mathbf{3}=\text { severa } \\
\text { Eritema }(E) \\
\text { Pápulas }(P) \\
\text { Vesículas }(V) \\
\text { Fisuras }(F) \\
\text { Descamación }(D) \\
\text { Edema }(E) \\
\text { SUM }(E+P+V+F+D+E)\end{array}$ & & & & & \\
\hline $\begin{array}{l}\text { Extensión de lesiones en ambas } \\
\text { manos (Ex) } \\
0=0 \% \\
1=1-25 \% \\
2=26-50 \% \\
3=51-75 \% \\
4=76-100 \% \\
\end{array}$ & & & & & \\
\hline $\begin{array}{l}\text { Total HECSI } \\
\text { Mínimo } 0 \\
\text { Máximo } 360\end{array}$ & SUM $\times$ Ex+ & SUM $\times$ Ex+ & SUM $\times$ Ex+ & SUM $\times$ Ex+ & SUM $x \mathrm{Ex}+$ \\
\hline
\end{tabular}


Además de las medidas preventivas, se deben instaurar programas de educación enfocados al cuidado y protección cutáneos, aportando conocimientos y mejorando las condiciones laborales.

El uso de cremas emolientes ricas en lípidos promueve la curación y previene nuevos episodios. Idealmente, deben estar libres de fragancias y contener pocos aditivos y conservantes, para evitar sensibilizaciones.

En caso de pacientes que deban lavarse las manos a menudo, se recomienda usar desinfectantes en base alcohólica con glicerina, menos irritantes que el agua y jabón.

El tipo de guantes se debe adaptar al tipo de trabajo y los compuestos que se manipulen. Conviene llevar los guantes durante el tiempo que sea necesario, pero tan corto como sea posible. Idealmente, se deben usar guantes de algodón debajo y cambiarlos cuando se humedezcan ${ }^{7}$.

\section{TRATAMIENTO}

El tratamiento del eccema de manos debe ajustarse a cada paciente, teniendo en cuenta la severidad, la evolución (aguda o crónica) y la morfología de las lesiones. En los casos de reciente comienzo, se debe tratar rápidamente para evitar la cronicidad.

Si la causa radica en la exposición a un alergeno o irritante conocido, el primer paso es evitarlo.

El tratamiento se aplica en forma escalonada y de acuerdo con el cuadro clínico (fig. 2):

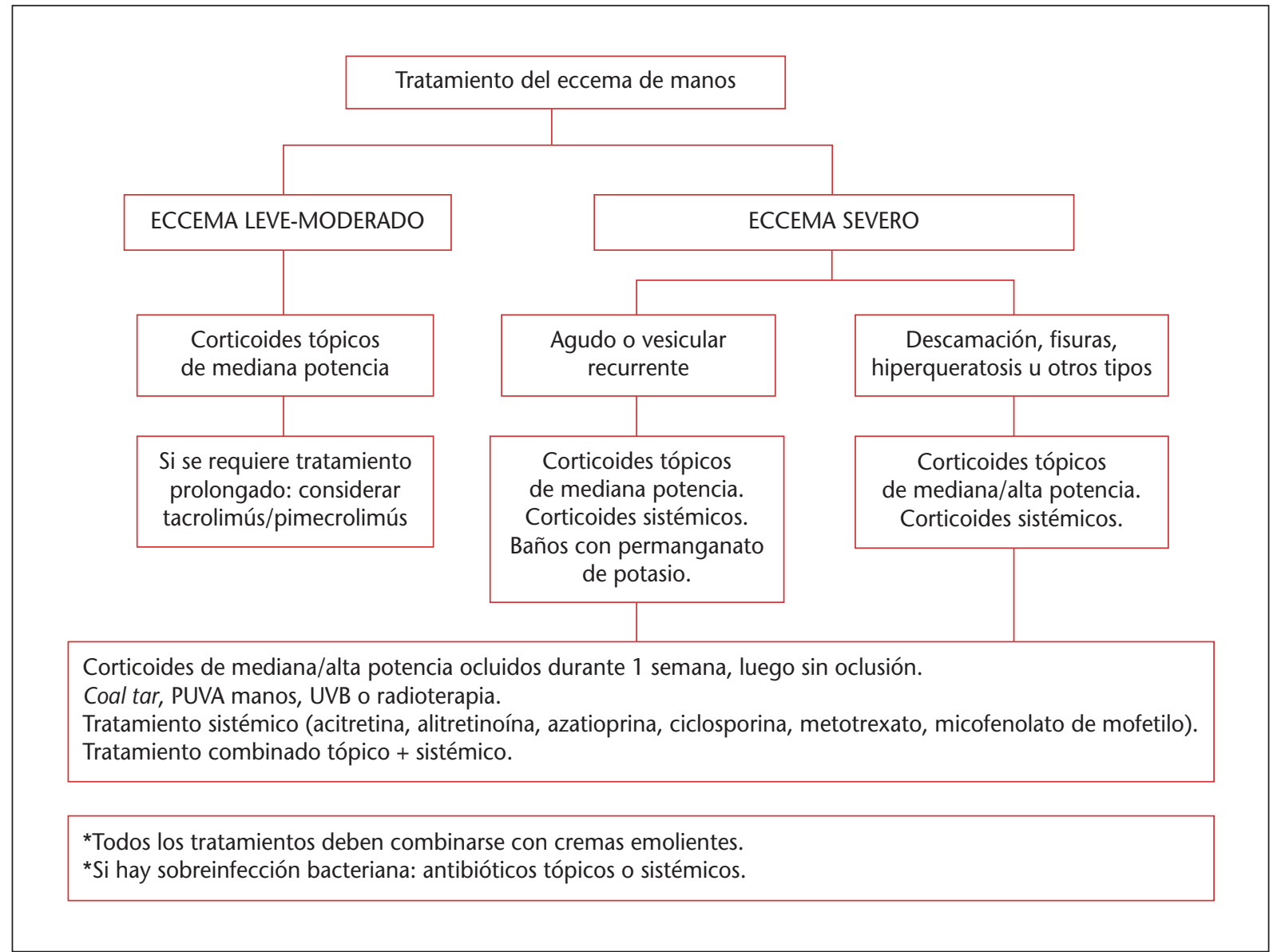

Figura 2. Algoritmo de tratamiento del eccema de manos.

Modificado de Menné et al. ${ }^{7}$. 
1. Tratamiento tópico:

- Emolientes.

- Corticoides de mediana o alta potencia (cremas, ungüentos).

- Inhibidores de la calcineurina tópicos (tacrolimús, pimecrolimús).

- Coal tary derivados.

- Fototerapia (PUVA manos, UVB, radiaciones ionizantes).

2. Tratamiento sistémico: se indica por períodos cortos. No hay resultados concluyentes en cuanto a dosis y eficacia en los estudios realizados.

- Acitretina $(25 \mathrm{mg} / \mathrm{d})$.

- Azatioprina.

- Alitretinoína (30 mg/d). No disponible en España a la fecha.

- Ciclosporina (2,5-3 mg/kg/d).

- Metotrexato (10-15 mg/semana).

- Micofenolato de mofetilo (2-3 g/d).

- Corticoides sistémicos (prednisona 0,5-1 mg/kg/d, prednisolona 20-40 mg/d).

La mayoría de los pacientes se controla con medidas de prevención y tratamiento tópico.

Los corticoides tópicos de mediana y alta potencia son el tratamiento de elección; se utilizan cada día durante un período determinado (2-4 semanas) y luego se pueden suspender o continuar (2-3 veces/semana) $)^{7}$.

Según la localización y evolución de las lesiones, elegiremos el tipo de corticoide y el vehículo adecuado para el paciente. Una localización palmoplantar requiere corticoides de potencia alta y muy alta, en ocasiones incluso en cura oclusiva.

Situaciones como calor, oclusión, erosiones, inflamación y estado de hidratación modifican la penetración de los corticoides, factores que deben tenerse en cuenta.

En el caso de lesiones liquenificadas y con fisuras, sería preferible utilizar corticoides de alta o muy alta potencia en un vehículo graso, como pomada o ungüento.

Si las lesiones son agudas o subagudas, se utilizan corticoides de mediana o alta potencia en forma de crema ${ }^{8}$.
El tiempo de aplicación de los corticoides debe limitarse en lo posible a unas 3 semanas en los casos de muy alta potencia.

Sin embargo, no existe tratamiento satisfactorio para el eccema de manos crónico severo (5-7\%) o el refractario al tratamiento tópico $(2-4 \%)^{9}$.

Los inhibidores de la calcineurina no poseen efectos adversos a largo plazo, aunque el efecto secundario más habitual es la sensación de quemazón en el área de aplicación. En el eccema de manos, se usan en los períodos más estables de la enfermedad, alternándolos con los corticoides cuando sea preciso.

A pesar de la amplia disponibilidad de tratamientos sistémicos, se ha visto que son capaces de inducir remisiones temporales, pero no llegan a controlar la enfermedad a largo plazo ${ }^{9}$.

\section{BIBLIOGRAFÍA}

1. Duus Johansen J, Hald M, Andersen BL, Laurberg G, Danielsen A, Avnstorp C, et al. Classification of hand eczema: clinical and etiological types. Based on the guideline of the Danish contact Dermatitis group. Contact Dermatitis. 2012:65:1321.

2. Thyssen JP, Johansen JD, Linneberg A, Menné T. The epidemiology of hand eczema in the general population-prevalence and main findings. Contact Dermatitis. 2010:62(2):75-87.

3. Veien N K, Menné T. Treatment of hand eczema. Skin Therapy letter.com (en línea). Consultado el 27/02/2012. Disponible en: http://www.skintherapyletter.com/2003/8.5/2.html

4. Van Gils RF, Boot C, van Gils PF, Bruynzeel D, Coenraads P J, Van Mechelen W, et al. Effectiveness of prevention programmes for hand dermatitis: a systematic review of the literature. Contact Dermatitis. 2011:64:63-72.

5. Agner T, Andersen KE, Brandao FM, Bruynzeel DP, Bruze M, Frosch $P$, et al. Hand eczema severity and quality of life: a crosssectional, multicentre study of hand eczema patients. Contact Dermatitis. 2008:59:43-7.

6. Held E, Skoet R, Johansen J D, Agner T. The hand eczema severity index (HECSI): a scoring system for clinical assessment of hand eczema. A study of inter- and intraobserver reliability. $\mathrm{Br}$ J Dermatol. 2005:152:302-7.

7. Menné T, Johansen JD, Sommerlund M, Veien NK. Hand eczema guidelines based on the Danish guidelines for the diagnosis and treatment of hand eczema. Contact Dermatitis. 2011:65:3-12.

8. Rodríguez Caruncho C, Ribera Pibernat M. Tratamiento del eccema de manos. Piel. 2009:24(9):505-12.

9. Diegpen TL, Agner T, Aberer W, Berth-Jones J, Cambazard F, Elsner P, et al. Management of chronic hand eczema. Contact Dermatitis. 2007:57:203-10. 